\title{
Analysis of a moving measurement platform based on line profile sensors for project- level pavement evaluation
}

Skar, Asmus; Levenberg, Eyal; Andersen, Sebastian; Andersen, Mathias B.

\section{Published in:}

Road Materials and Pavement Design

Link to article, DOI:

$10.1080 / 14680629.2020 .1741429$

Publication date:

2021

Document Version

Peer reviewed version

Link back to DTU Orbit

Citation (APA):

Skar, A., Levenberg, E., Andersen, S., \& Andersen, M. B. (2021). Analysis of a moving measurement platform based on line profile sensors for project-level pavement evaluation. Road Materials and Pavement Design, 22(9), 2069-2085. https://doi.org/10.1080/14680629.2020.1741429

\section{General rights}

Copyright and moral rights for the publications made accessible in the public portal are retained by the authors and/or other copyright owners and it is a condition of accessing publications that users recognise and abide by the legal requirements associated with these rights.

- Users may download and print one copy of any publication from the public portal for the purpose of private study or research.

- You may not further distribute the material or use it for any profit-making activity or commercial gain

- You may freely distribute the URL identifying the publication in the public portal 


\title{
RESEARCH ARTICLE
}

\section{Analysis of a moving measurement platform based on line profile sensors for project-level pavement evaluation}

\author{
Asmus Skar ${ }^{\mathrm{a}}$, Eyal Levenberg ${ }^{\mathrm{a}}$, Sebastian Andersen ${ }^{\mathrm{a}}$ and Mathias B. Andersen ${ }^{\mathrm{b}}$ \\ ${ }^{a}$ Technichal University of Denmark, Department of Civil Engineering, Nordvej, Building 119, \\ 2800 Kgs. Lyngby, Denmark; ${ }^{\text {b}}$ FOSS, Nils Foss Allé 1, 3400 Hillerød, Denmark
}

\begin{abstract}
ARTICLE HISTORY
Compiled January 29, 2020

ABSTRACT

In recent years there has been renewed interest in developing Moving Measurement Platforms (MMPs) to replace the Falling Weight Deflectometer (FWD) which currently serves as the industry standard for project-level pavement evaluation. The purpose of this study was to contribute to the transition efforts from FWD to MMPs. While focusing on a new MMP device (called Raptor) based on line profile sensing technology, the work addressed three fundamental aspects needed for eventual field data assessment and industry acceptance: $(i)$ loading and measurement setup - presenting an innovative device consisting of a beam equipped with several line profilers and also motion tracking sensors. By means of image analysis this configuration allows for tracking a point on the pavement surface and measuring displacement information while moving. (ii) interpretation method - capable of accepting devicemeasured data as input, and providing elastic properties for a layered pavement model. (iii) sensitivity to measurement errors - where the interpretation method is first validated and then interrogated for error effects on the inferred moduli. Overall, the obtained results provide basic confidence in the device and the interpretation method, and demonstrate that the technology has the potential to meet project-level pavement evaluation needs.
\end{abstract}

\section{KEYWORDS}

Inverse analysis; Monte Carlo simulation; Moving measurement platform; Raptor; Sensitivity analysis; Pavement analysis

\section{Introduction}

The Falling Weight Deflectometer (FWD), introduced in the 1970s (Ullidtz, 1987), is currently regarded as the device of choice for performing non-destructive mechanical evaluation of pavements (ASTM-D4694; ASTM-D4695). The FWD is routinely operated at the network-level for supporting pavement management decisions; doing so is based on calculating structural condition indices from measured deflection peaks (Horak, Hefer, Maina, \& Emery, 2015; Kilareski \& Anani, 1982). The device is also routinely employed at the project-level for backcalculation of layer properties. For this purpose the pavement system is typically modeled as a weightless stratified linear elastic half-space having a priori known layer thicknesses and interface conditions (SPT1026; SPT1198; SPT1375). Although more advanced interpretation schemes exist 
(see e.g., Chatti, Ji, and Harichandran (2004); Madsen and Levenberg (2018); Varma, Kutay, and Levenberg (2013)), none have yet to gain widespread engineering acceptance similar to the elastostatic backcalculation.

The FWD achieved its industry-standard status after several decades of exhaustive research that targeted device improvements and scientific understanding of three fundamental aspects: $(i)$ loading and measurement setup, e.g. mechanical design, loading mechanism, sensing technologies, and data acquisition (L. H. Irwin \& Richter, 2005), (ii) data interpretation methods, e.g., forward modeling and simplifying assumptions, inverse analysis schemes, and computational efficiency considerations (Khazanovich, Tayabji, Darter, \& ERES Consultants, 2001; Von Quintus \& Simpson, 2002), and (iii) sensitivity to measurement errors, i.e., quantifying how backcalculated results are influenced by, e.g., finite sensing resolution, incorrect sensor spacing, and erroneous layer thicknesses (L. Irwin, Yang, \& Stubstad, 1989; Sangghaleh et al., 2014; Siddharthan, Sebaaly, \& Javaregowda, 1996; Stubstad, Irwin, Lukanen, \& Clevenson, 2000).

Performing pavement structural evaluation from a Moving Measurement Platform (MMP) holds considerable advantages over the stationary FWD. The three main benefits include: $(i)$ improved operational safety - as there is no need for lane closures, (ii) enhanced spatial coverage - given the superior testing efficiency, and (iii) exciting the pavement in a manner that is representative of service conditions - due to a rolling wheel type-of-loading.

It appears that present-day computing powers and sensing technologies are advanced to the point that allow building such platforms, first and foremost for pavement management applications (Elseifi, Gaspard, Wilke, Zhang, \& Hegab, 2015; Flintsch et al., 2013; Katicha, Flintsch, \& Ferne, 2013; Levenberg, Pettinari, Baltzer, \& Christensen, 2018; Muller \& Roberts, 2013; Nasimifar, Siddharthan, Hajj, \& Motamed, 2016; Zofka, Sudyka, Maliszewski, Harasim, \& Sybilski, 2014). At the same time the technology is rapidly approaching project-level capabilities of inferring individual elastic layer properties (Andersen, Levenberg, \& Andersen, 2017; Nasimifar, Thyagarajan, \& Sivaneswaran, 2017). Current MMP devices include the Lacroix-LPC deflectograph (Gressin, 1982), the Euroconsult Curviameter (J.-M. Simonin, Geffard, \& Hornych, 2015), the Greenwood Traffic Speed Deflectomenter (TSD) (Pedersen, 2013; J. Simonin, Lièvre, Rasmussen, \& Hildebrand, 2005), the ARA Rolling Wheel Deflectomenter (RWD) (Wilke et al., 2015), and the Dynatest Rapid Pavement Tester (Raptor) (Andersen et al., 2017).

Irrespective of device selected for pavement analysis, a model guided data processing approach is required for performing project-level interpretation. Thus, the procedure involve some pre-defined idealizations and assumptions that eventually will affect the interpretation method results, and how well they reflect the reality. Moreover, the model itself may be prone to errors and instability issues (see e.g. Garbowski and Pozarycki (2017); Goktepe, Agar, and Lav (2006); Sivaneswaran, Kramer, and Mahoney (1991)). Thus, any data interpretation method, including forward modeling and inverse analysis schemes, should be tested and validated prior to assessment of field data

The purpose of this study is to contribute to the transition efforts from FWD to MMPs. Unlike previous work that focused on either the RWD (Briggs, Johnson, Stubstad, \& Pierce, 1999; Elbagalati, Elseifi, Gaspard, \& Zhang, 2017; Elseifi et al., 2015; Herr, Hall, White, \& Johnson, 1995; Wilke et al., 2015) or the TSD (Elbagalati, Mousa, Elseifi, Gaspard, \& Zhang, 2018; Flintsch et al., 2013; Graczyk, Zofka, Sudyka, \& Rafa, 2014; Katicha et al., 2013; Levenberg et al., 2018; Manoharan, Chai, Chowdhury, \& Golding, 2018; Muller \& Roberts, 2013; Nasimifar et al., 2016; Nasimifar, 
Siddharthan, Rada, \& Nazarian, 2017; Zofka, Graczyk, \& Rafa, 2015; Zofka et al., 2014), this contribution is focused on the Raptor. The work adresses the three fundamental aspects needed for eventual field data assessment and industry acceptance. Initially presented is the device's loading and measurement setup. Presented next is an inverse analysis method for performing project-level interpretation, i.e., resolving elastic layer properties from the collected readings. Thirdly, for the given setup and interpretation method, a synthetic investigation is carried out to validate the approach and quantify the effects of measurement errors on inferred layer moduli.

The synthetic methodology proposed has the important advantage that the target layer moduli are known, enabling basic model validation. It also has the advantage of resolving the difficulty of uncoupling the effects systematic and random measurement errors have on the inferred moduli (Sangghaleh et al., 2014). This allows for identification of critical device components and assessment of the overall robustness of the method. Finally, the effects from random measurement errors on the interpretation method results can be quantified prior to field experiments. This can be utilized by manufacturers to optimize the design and tailor the device setup dependent on application.

\section{Loading and Measurement Setup}

\subsection{Device overview}

Many MMPs for pavement evaluation are constructed following the original design ideas of Harr (M. Harr \& Ng-A-Qui, 1977; M. E. Harr \& Elton, 1983). Accordingly, platforms consist of a heavy wheeled vehicle equipped with a long stiff beam installed in the travel direction. The beam is instrumented with sensors that are expected to provide information about surface displacements generated by the MMP tires. It is assumed that the sensors are rigidly attached to the beam and therefore mechanically constrained to move together.

The Raptor device is a semi-trailer truck equipped (at the rear) with two independent single-wheel suspension systems - one on each side. Its sensor beam is installed in an environmentally protected compartment at the bottom of the platform, approximately $200 \mathrm{~mm}$ above the pavement surface, passing close to one of the tires (from the inner side); it carries an array of laser-based line profilers. Figure 1 shows a sketch of the Raptor measurement trailer and truck with a sensor beam carrying $I$ lasers $(i=1 . . I)$ arranged such that Laser $I$ is the forwardmost laser and Laser 1 is the rearmost laser. The forces in the figure represent static axle loads with $V$ denoting the travel speed. A plan view of the platform is offered in Figure 1 (a) and a side view is shown in Figure 1 (b). The designation $2 \times 50 \mathrm{kN}$ is used as there is no connection between the rear wheels. The shape of the deformed pavement surface for a cross-section taken directly below the sensor beam (i.e., along $x$ with $y=0$ ) is also sketched in Figure 1 (b). This sketch was prepared to provide intuition on the expected laser readings; it is based on a linear elastic half-space.

\subsection{Principle of operation}

Each of the lasers carried by the Raptor sensor beam measures self-distance to the pavement surface along a line that is oriented transversely to the travel direction. This line is referred to herein as 'detection window' (see Figure 2 (a)); it is approximately 
(a)
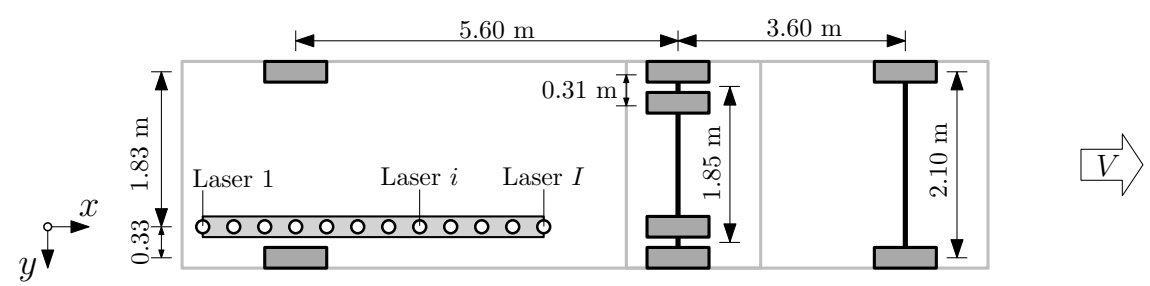

(b)

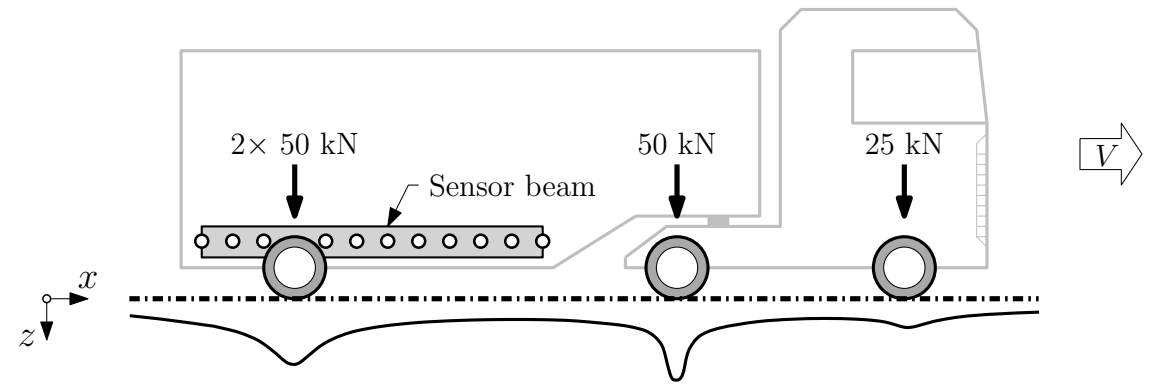

Figure 1. Sketch of the Raptor MMP truck and trailer: (a) top view x-y plane and (b) side view x-z plane. The dash dotted line marks the undeformed pavement surface, and the solid line marks the deformed pavement surface.

$200 \mathrm{~mm}$ long composed of over 1000 individually measured distance points, each about 200 micrometer in diameter. With a sample rate of about $4000 \mathrm{~Hz}$, a single laser line profiler produces about four million data points per second. When all measurements from a single laser are placed sequentially like rows in a matrix, a three-dimensional scan of the road surface transpires as the Raptor moves forward. This is schematically shown in Figure $2(\mathrm{~b})$ for lasers $i$ and $i-1$. The individual distance points are denoted by pixels, with a greyscale shading that corresponds to the measured distance values. The outcome appears noisy and erratic because the lasers are sensitive enough to also map the surface texture.

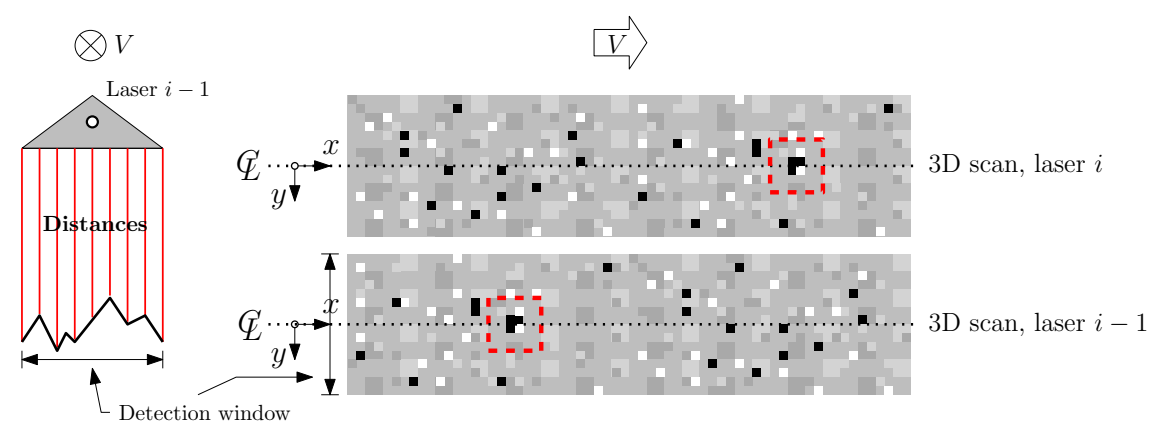

(a)

(b)

Figure 2. Sketch of line laser and distance to surface measured: (a) Cross-section of line laser $i-1$ showing the detection window (b) a planar view of the $3 \mathrm{D}$ surface scan used for image analysis. The relative difference between distances measured from the two lasers are shown as grayscale pixels.

As the Raptor moves forward in a straight line, any random point on the pavement surface that enters the detection window of the forwardmost sensor (Laser $I$ ) can be identified in the readings of all other lasers. The identification is achieved by means of an image correlation technique that matches texture patches encapsulating (separately for each laser) the random point being evaluated. In Figure 2 (b) two such patches are denoted by dashed square frames; these are $64 \times 64$ pixels in size. Given that the natural texture surrounding a point at the pavement surface is unique, the 
identification of a patch across all scans is achieved with a high level of confidence. Having a detection window that is $200 \mathrm{~mm}$ long provides compensation for beam roll or yaw rotations (or both); it ensures that the sought patch remains inside the detection boundaries across all lasers. Conversely, if these rotations are independently measured, then the patch-matching idea can be utilized to also identify sideway displacements of the point being tracked. This latter option is not addressed at this time; herein, all surface displacements in the $y$-axis direction are not considered for analysis. The length of the detection window also provides compensation for the Raptor not moving in a perfectly straight line.

\subsection{Basic measurement data}

The Raptor sensor beam is additionally instrumented with motion tracking sensors such as gyroscopes and accelerometers. For short time intervals, and by way of integration, these sensors enable the tracking of changes in beam pitch angle and height. To best illustrate the type of data being collected during a standard Raptor operation, only a portion of the sensor beam is considered - hosting two lasers $i$ and $i-1$ and moving at a constant speed $V$ (see Figure 3 ). In this figure the unloaded pavement surface (before deformation) is marked by a straight dashed dotted line. A right-handed Cartesian coordinate system that moves with the beam is located with the origin at the unloaded surface; the $x$-axis is oriented along the travel direction and the $z$-axis is pointing down into the medium. The loaded pavement surface (after deformation) is marked by a curved solid line. Solely for graphical clarity the surface is depicted as smooth; in reality, the surface has some texture which is rough at both the micro and macro scales. Such roughness has no consequence because the principle of operation is based on detecting points.
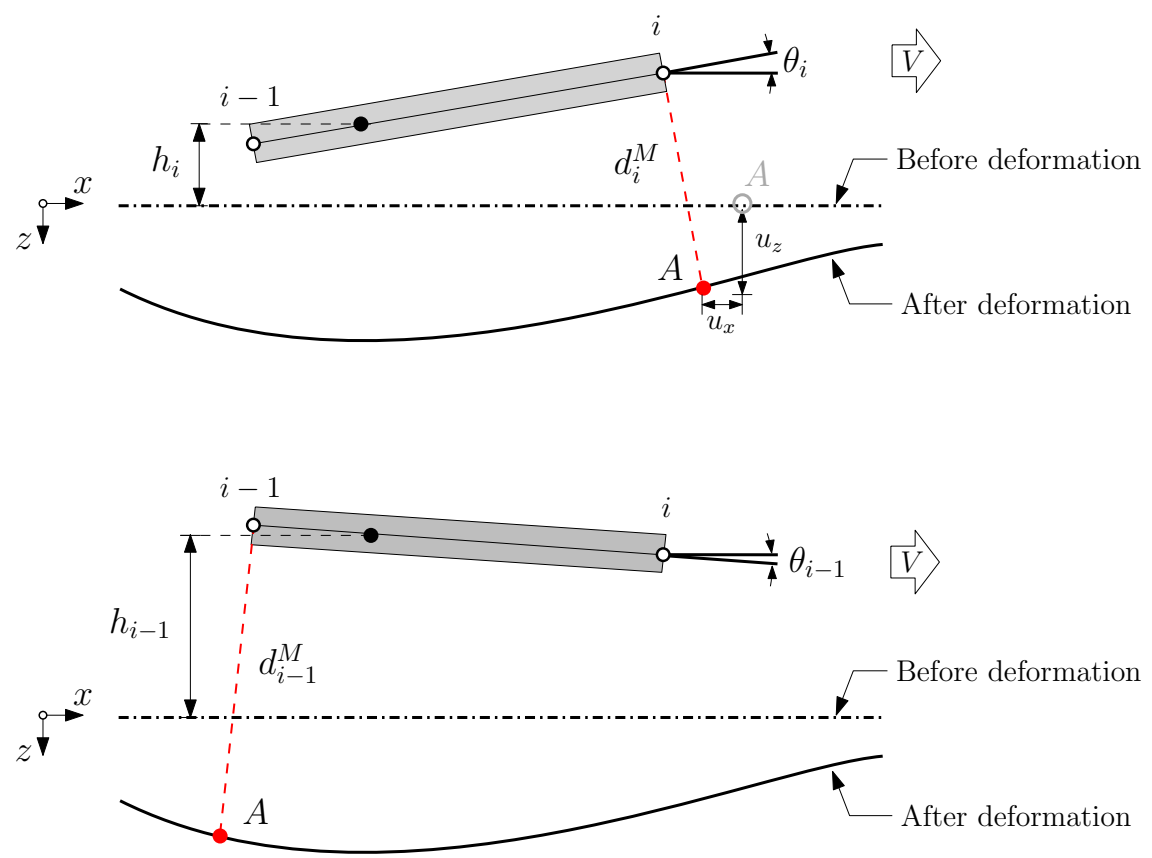

Figure 3. Illustration of measurment data provided by the Raptor device in side view (x-z plane): (a) distance $d_{i}^{M}$ and (b) distance $d_{i-1}^{M}$ at a later time. The point $A$ is shown in undeformed (gray) and deformed (red) state. 
As shown in Figure 3 (a) laser $i$ measures a distance $d_{i}^{M}$ to some random evaluation point $A$ on the pavement surface. When laser $i$ detects point $A$ the angle of the beam is $\theta_{i}$ and the height is $h_{i}$ (to some reference point on the beam). The displacements of the point in the $x-z$ plane are denoted by $u_{x}$ and $u_{z}$. This is to illustrate that two orthogonal displacements are considered. At a later time, Figure 3 (b), laser $i-1$ measures the distance $d_{i-1}^{M}$ to the same point $A$. The corresponding beam angle is $\theta_{i-1}$ and the height is $h_{i-1}$.

Thus, the basic measurement data provided by the Raptor device for a random evaluation point includes: distance readings from each of the lasers $d_{i}^{M}$ 's, change in sensor beam angle $\Delta \theta_{i \rightarrow i-1}$ and change in sensor beam height $\Delta h_{i \rightarrow i-1}$ in-between consecutive detections (from the motion tracking sensors), where $\Delta \theta_{i \rightarrow i-1}=\theta_{i-1}-\theta_{i}$ and $\Delta h_{i \rightarrow i-1}=h_{i-1}-h_{i}$.

\section{Data interpretation method}

This section describes a method for performing project-level interpretation based on Raptor data. The method consists of three elements: $(i)$ a mechanical pavement model, (ii) a Raptor simulation module, and (iii) an optimization algorithm. The mechanical pavement model is chosen to be the classic formulation for an $N$-layered half-space (Burmister, 1945; Huang, 2004; Ioannides \& Khazanovich, 1998). All layers are assumed linear elastic, isotropic, homogeneous, fully bonded, and weightless.

The model inputs include Youngs modulus $E_{n}$, Poissons ratio $\nu_{n}$, and layer thickness $t_{n}$ (where $n$ denotes the layer number). This model is engaged to calculate the surface displacements $u_{x}$ and $u_{z}$ (see Figure 3 (a)) at any point of interest and for a given set of loading conditions. To improve computational effciency and accuracy during recalculation of surface displacements the methodology proposed in Andersen, Levenberg, and Andersen (2018) was employed. In principle there is no restriction in the data interpretation method for using a more advanced mechanical pavement model (e.g., including viscoelastic material behavior, interlayed debonding effects, etc.). The only concern with higher order models is the ability to reliably identify the governing parameters based on the measured data.

The Raptor simulation module involves loading the pavement model according to the Raptor wheel configuration (see Figure 1), and then forward-simulating the measured distances to a random evaluation point from a virtual sensor beam. For this purpose all pavement model parameters are either known or assumed, along with the pitch angle $\theta_{I}$ and the height $h_{I}$ of the sensor beam when the point is first detected (by laser $I$ ). All subsequent beam angles and heights needed for the forward-simulation are based on the measured $\Delta \theta_{i \rightarrow i-1}$ and $\Delta h_{i \rightarrow i-1}$ as follows

$$
\begin{array}{ll}
\theta_{i}=\theta_{I}+\sum_{j=i+1}^{j=I} \Delta \theta_{j \rightarrow j-1} & (j \leq I) \\
h_{i}=h_{I}+\sum_{j=i+1}^{j=I} \Delta h_{j \rightarrow j-1} & (j \leq I)
\end{array}
$$

The output of this module is forward simulated distance readings $d_{i}^{F S}$ 's corresponding to the measured distance readings $d_{i}^{M}$ 's. More details on associated calculations can 
be found in Andersen et al. (2017).

The third interpretation element (optimization algorithm) deals with formulating and solving an inverse problem. Specifically, the problem is to find the $E_{n}$ 's in the Raptor module such that a best match is achieved between all measured and forward simulated distance readings. This task is cast as a minimization problem:

$$
E_{n}^{*}=\underset{E_{n}, \theta_{I}, h_{I}}{\operatorname{argmin}} \psi
$$

where $\psi$ is an objective function that embodies the mismatch between measured and forward-simulated distances, i.e., $\psi=\psi\left(d_{i}^{M}, d_{i}^{F S}\right)$, and $E_{n}^{*}$ are the $E_{n}$ 's that minimize $\psi$. The parameters $\theta_{I}$ and $h_{I}$ are included in the minimization because they affect $\psi$ and cannot be directly obtained from the device instrumentation. The numerical values of the optimal $\theta_{I}$ and $h_{I}$ are considered as a by-product of the interpretation method given that they are not useful to pavement engineering applications (e.g., they reflect vehicle characteristics). All other parameters governing $\psi$, e.g., layer thicknesses, Poisson's ratios, Raptor loading configuration, and angle and height changes of the sensor beam, are taken as known.

It is herein proposed to base the objective function formulation on weighted absolute differences, as follows:

$$
\psi=\frac{1}{I} \sum_{i=1}^{I}\left|d_{i}^{M}-d_{i}^{F S}\right| \cdot w_{i}
$$

where $w_{i}$ 's are unitless weights introduced to account for (expected) dissimilarities in pavement displacement magnitudes below the sensor beam. So doing this is needed for balancing the objective function, i.e., ensuring that laser distances (either measured or forward-simulated) taken closer to the Raptor tires, where the pavement surface displacements are larger, do not diminish the influence of those taken further away - where the displacements are smaller. Herein, these weights are chosen assuming an elastic half-space, as the relative vertical displacements compared to the laser closest to the load at the different laser positions (see Figure 1 (b)). In this connection, attempting to base the formulation of $\psi$ on relative errors (between measured and forward-simulated) is deemed ineffective because these are dominated by the height of the sensor beam above the pavement surface.

\section{Validation and error sensitivity}

\subsection{Methodology}

Based on the aforementioned device setup and data interpretation method, investigated next is the ability to correctly infer layer moduli in a given situation. The chosen methodology for addressing this matter is pursued here with measured distances that are synthetically generated from a model and not obtained from a field experiment. This approach has an important advantage that the target layer moduli are known. It also has the advantage of resolving the difficulty of uncoupling the effects systematic and random measurement errors have on the inferred moduli (Sangghaleh et al., 2014). 
Subsequently the investigation is carried out in three separate efforts: $(i)$ validating that the interpretation method yields the correct solution in a perfect situation, i.e., when no measurement errors are present and when all interpretation assumptions are correct, (ii) assessing the sensitivity to systematic errors, representing an incorrectly placed laser (on the sensor beam), or working with an uncalibrated motion tracking sensor, and ( $\mathrm{iii}$ ) assessing the sensitivity to random errors, representing noisy data or limited measurement resolution.

\subsection{Synthetic data generation}

In order to generate synthetic data for subsequent interpretation the loading configuration shown in Figure 1 was assumed, with all tire-pavement contact areas taken as circular having a radius of $150 \mathrm{~mm}$. Also, based on the first generation Raptor device, a $5.1 \mathrm{~m}$ long sensor beam was chosen, housing 12 unevenly spaced lasers (i.e. $I=12$ ), with Laser 5 being the closest to the center of the rear right wheel of the trailer (offset of $0.33 \mathrm{~m}$ ). The sensor positions along the beam (in meters) are: $-1.5,-0.9,-0.6$, $-0.3,0.0,0.3,0.6,0.9,1.5,1.8,3.0,3.6$. The corresponding objective function weights $w_{1}, . ., w_{12}$ in Equation 3 are: 3.04, 2.20, 1.74, 1.27, 1.00, 1.26, 1.70, 2.12, 2.80, 3.07, 3.72 and 3.72 .

Two different pavement systems were defined for evaluating the interpretation method (see Figure 4) based on the current Danish pavement design guidelines (Baltzer et al., 2017). System 1 corresponds to a heavily trafficked highway and System 2 to a lightly trafficked roadway. To indicate reference (i.e., target or correct) moduli, material properties and layer thicknesses are as shown with the added superscript 0 .

As can be seen, a 2-layered system is utilized, with the upper layer (Layer 1) representing the pavement structure (i.e., the combined thicknesses and moduli of unbound subbase, base, and asphalt concrete), and the half-space (Layer 2) representing the subgrade and deeper soil layers. One reason for choosing to work with 2-layered systems is the ability to graphically display the objective function (Equation 3) in the modulus space, and thereby illustrate the existence of a clear minimum. Another reason is related to the minimal sensor spacings assumed above. Along the sensor beam the minimal laser spacing is $300 \mathrm{~mm}$; also, Laser 5 (closest to the load) is offset by 333 $\mathrm{mm}$ from the center of the nearby rear Raptor wheel. These spacings practically limit the ability to correctly infer moduli of thin pavement layers (Chou \& Lytton, 1991; L. Irwin et al., 1989; Mehta \& Roque, 2003; Uzan, 1994). Increasing the sensitivity to thinner pavement layers may be achieved by reducing laser spacings or minimizing the distance between the beam and the Raptor wheel (or both). However, doing this will also require that the overall measurement capabilities can compliment the closer spacings. 


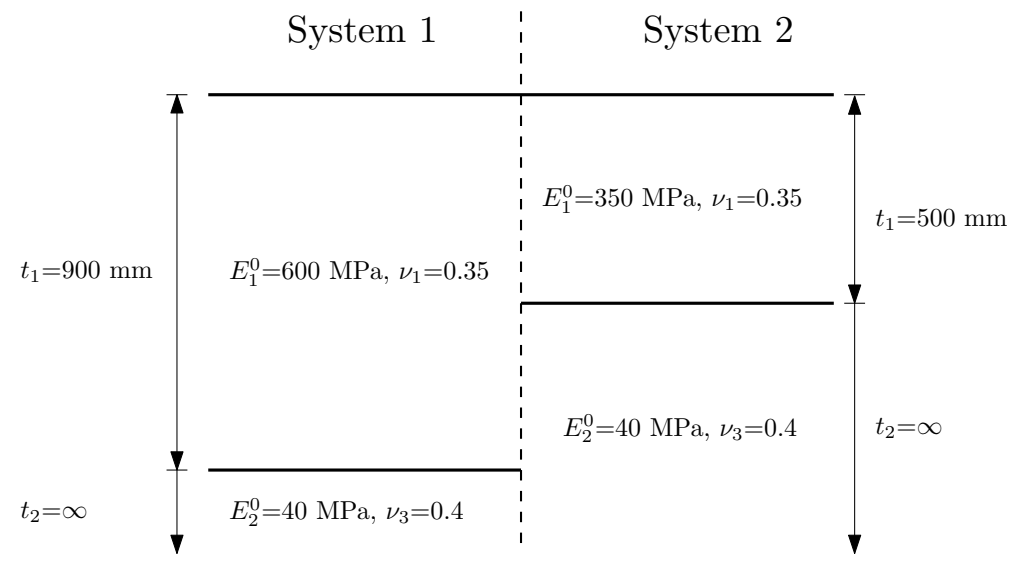

Figure 4. Pavement material- and geometrical properties for generating synthetic data used in validation and sensitivity analysis.

For a random evaluation point, synthetic measurement data were separately generated for the two pavement systems in Figure 4. This was done assuming an initial beam angle $\theta_{I}=0.001 \mathrm{rad}$, an initial beam height $h_{I}=176.98 \mathrm{~mm}$, and subsequent angle and height changes as shown in Table 1. The resulting 'measured' distances, associated with pavement Systems 1 and 2, are referred to (in Table 1) as Case 1 and 2 (respectively).

Table 1. Synthetically generated input data.

\begin{tabular}{|c|c|c|c|c|}
\hline & & & Case 1 & Case 2 \\
\hline Laser & Angle change & Height change & \multicolumn{2}{|c|}{ Distances } \\
\hline$i$ & $\Delta \theta_{i \rightarrow i-1}$ & $\Delta h_{i \rightarrow i-1}$ & $d_{i}^{M}$ & $d_{i}^{M}$ \\
\hline$[-]$ & {$[\mathrm{rad}]$} & {$[\mathrm{mm}]$} & {$[\mathrm{mm}]$} & {$[\mathrm{mm}]$} \\
\hline 1 & - & - & 165.6510 & 165.6727 \\
\hline 2 & +0.0020 & +2.00 & 174.0215 & 174.1259 \\
\hline 3 & +0.0030 & +0.20 & 177.7072 & 177.8768 \\
\hline 4 & -0.0060 & -1.20 & 175.5020 & 175.7402 \\
\hline 5 & +0.0040 & -2.50 & 175.5755 & 175.8527 \\
\hline 6 & +0.0030 & +2.60 & 178.6033 & 178.8405 \\
\hline 7 & -0.0040 & -1.30 & 180.1245 & 180.2913 \\
\hline 8 & -0.0010 & +1.10 & 183.7462 & 183.8482 \\
\hline 9 & +0.0030 & +0.10 & 183.1890 & 183.2062 \\
\hline 10 & +0.0010 & -3.10 & 179.3150 & 179.3090 \\
\hline 11 & -0.0035 & +2.30 & 195.0594 & 195.0294 \\
\hline 12 & +0.0050 & +0.30 & 180.9489 & 180.9308 \\
\hline
\end{tabular}

\subsection{Model validation}

The analysis in this subsection focuses on the ideal scenario, where the synthetic data in Table 1 are utilized as input. Specifically, the purpose is to ensure that the proposed objective function (Equation 3) can offer favorable convergence conditions for an optimization algorithm. Such analysis is a necessary first step before introducing 
errors to the synthetic data and assessing sensitivities to imperfect inputs.

The graphical behavior of the objective function in the vicinity of the reference solution is shown in Figure 5. This Figure contains four charts, such that Figures 5 (a) and (b) are related to Case 1 (System 1 pavement) and Figures 5 (c) and (d) are related to Case 2 (System 2 pavement). Figures 5 (a) and (c) depict contour plots of $\psi$ in the (normalized) modulus space. The planar axes are unitless, each denoting the ratio between forward-simulated moduli $\left(E_{n}^{F S}\right.$ 's) and their corresponding reference moduli $\left(E_{n}^{0}\right.$ 's). The applicate axes designate objective function values; these are color coded with darker shading indicating larger $\psi$ values. For both cases it can be noticed that there is a clear minimum in vicinity of the correct solution (indicated by a red filled circular marker); the lightest shading appears where the modulus ratios are unity.

To further demonstrate the behavior of the objective function near the correct solution, cross-sections in two perpendicular directions, denoted $A-A$ and $B-B$ (indicated by the white dashed lines) are presented in Figures 5 (b) and (d). As can be seen, the objective function appears more sensitive to relative changes in $E_{2}^{F S}$ than to relative changes in $E_{1}^{F S}$. Nonetheless, the minimum of $\psi$ is very pronounced, indicating that it should be easily detected with any general-purpose nonlinear optimization algorithm. 


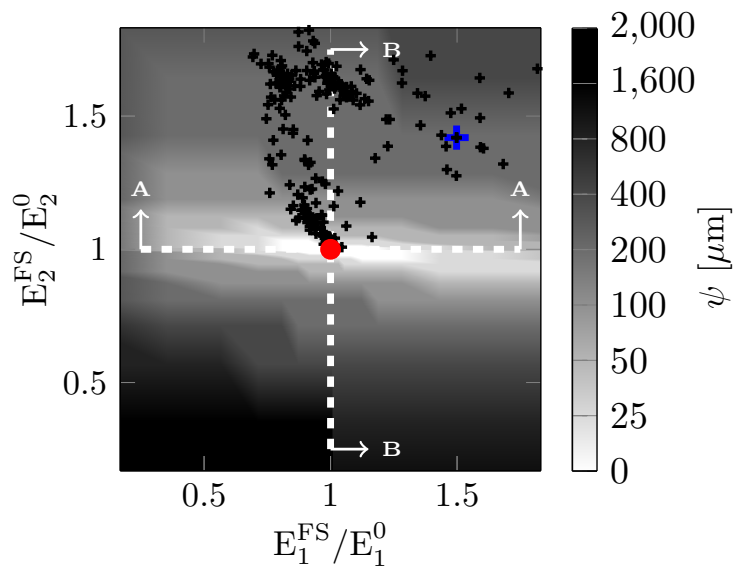

(a)

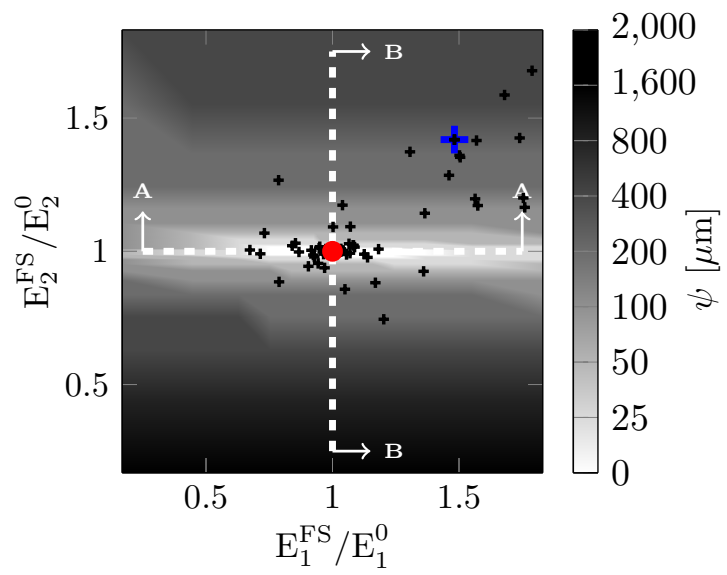

(c)

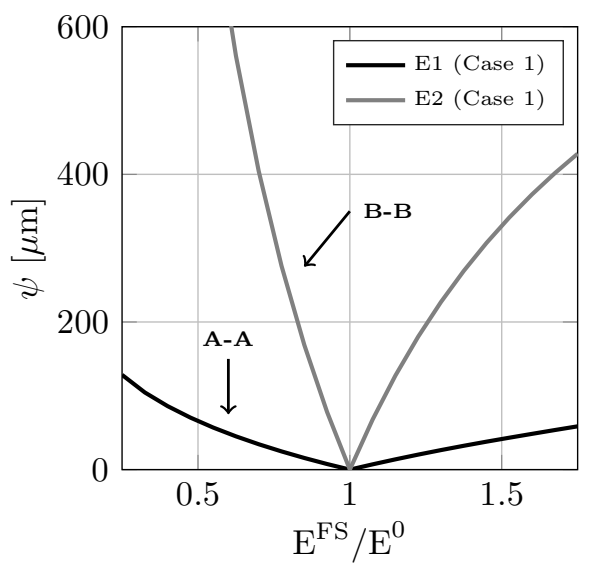

(b)

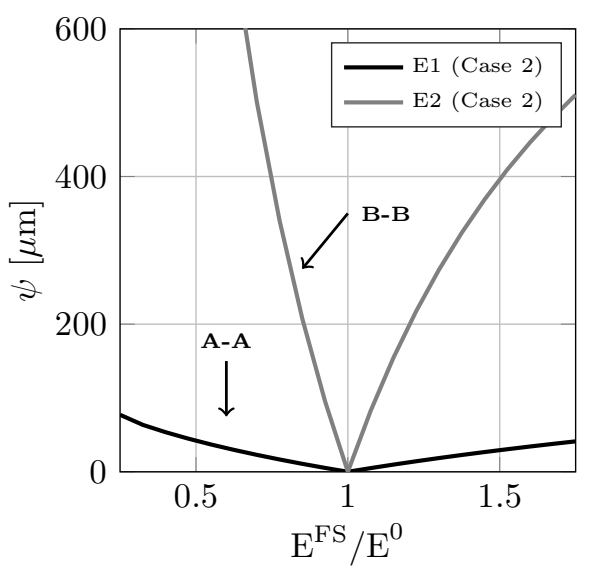

(d)

Figure 5. Plot of objective function as a function of $E_{1}^{F S}$ and $E_{2}^{F S}$ for the two layers; (a) and (b) representing Case 1 with a thick pavement section and (c) and (d) representing Case 2 with a thin pavement section. The initial moduli is indicated with a blue cross, intermidiate iterations with black crosses, and the optimal solution with a red filled circular marker.

An attempt was made to solve the minimization problem in Equation 2 for Cases 1 and 2 (separately) utilizing the Nelder-Mead simplex (NMS) multidimensional optimization algorithm (Nelder \& Mead, 1965). The NMS algorithm belongs to a class of direct search methods that operate with objective function values, without relying on derivative information. This algorithm is commonly applied in engineering problems, with reported success in performing elastodynamic backcalculation of FWD measurements (Yi \& Mun, 2009). The algorithm and search parameters are listed in Table 2. The latter were linearly scaled or transformed (or both) in order to arrive at an approximate magnitude of unity. Magnitude similarity among search parameters can help accelerate convergence, and aid in chosing a single uniform minimum parameter step for governing the algorithm. 
Table 2. Solution technique applied in the present study.

\begin{tabular}{ll}
\hline Solver technique & NMS \\
Min. function step (stop criterion) & $1 \cdot 10^{-4}$ \\
Min. parameter step (stop criterion) & $1 \cdot 10^{-4}$ \\
Max. iterations (stop criterion) & $1 \cdot 10^{3}$ \\
Moduli scaled, $\tilde{E}_{n}$ & $\log \left(E_{n}[\mathrm{MPa}] \cdot 10^{6}\right) / 8$ \\
Initial height scaled, $\tilde{h}_{I}$ & $h_{I}[\mathrm{~mm}] \cdot 6 / 1000$ \\
Initial angle scaled, $\tilde{\theta}_{I}$ & $\theta_{I}[\mathrm{rad}] \cdot 1000$ \\
\hline
\end{tabular}

Ultimately, the correct moduli values were inferred from the synthetic data for a wide range of initially assumed parameter values. This outcome provides basic confidence in the proposed method. An example convergence path is graphically presented in Figures 5 (a) and (c) where individual iteration results are depicted (with small black crosses) for an optimization process initialized at the point: $E_{1}^{F S}=1.5 E_{1}^{0}$ and $E_{2}^{F S}=1.4 E_{2}^{0}$.

\subsection{Sensitivity to systematic errors}

This subsection investigates the effects of systematic errors in the inputs to the interpretation method on the inferred layer moduli. Systematic errors, generically denoted here as $\epsilon^{\mathrm{s}}$, refer to a situation where an incorrect sensor reading is undetected beforehand, and subsequently treated as correct. This situation should of course be intercepted by a calibration procedure - ensuring that all lasers are aligned and reading correctly. Nonetheless, the proposed investigation is needed in order to identify critical device components (along with their subsequent calibration needs), as well as to assess method stability and overall robustness.

As a first step, 'measured' $d_{i}^{M}$ 's in Table 1 were individually offset (one at a time) by adding or subtracting $10 \mathrm{~mm}$ as to represent a single faulty laser reading. Nonetheless, this did not affect the interpretation method results; i.e., the optimization process converged to the correct (reference) layer moduli. In the second step, systematic errors were introduced to the 'measurements' originating from the motion tracking sensors that provide the angle and height changes of the sensor beam. Referring to Table 1, errors were introduced by adding or subtracting one percent to all the $\Delta \theta_{i \rightarrow i-1}$ 's and separately to all the $\Delta h_{i \rightarrow i-1}$ 's. These systematic errors did affect the interpretation outcome in the two considered cases (Table 1). The influence on Layer 1 and Layer 2 moduli is plotted in Figure 6 (a) and (b), respectively. The plus and minus signs in the plots indicate whether the difference is due to a positive or negative one percent offset error. 


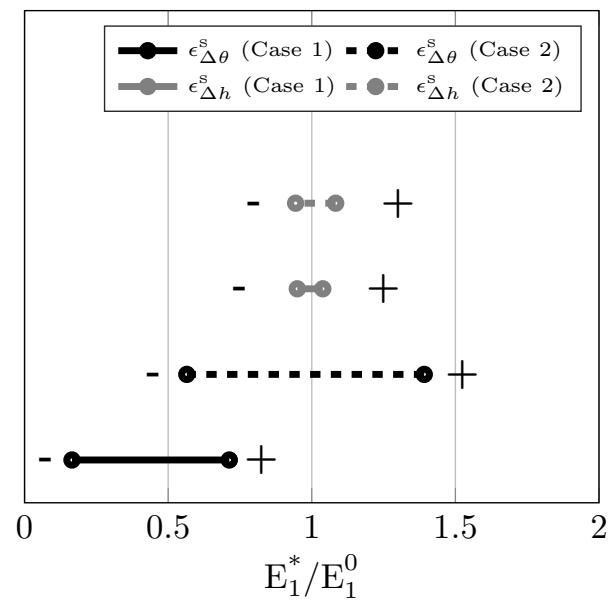

(a)

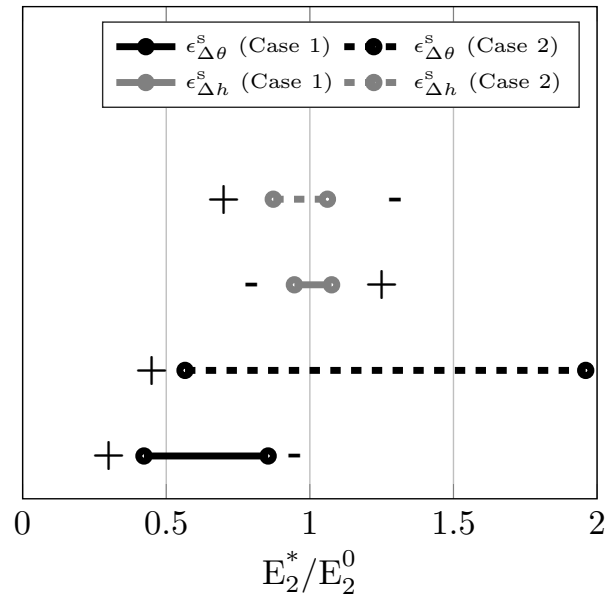

(b)

Figure 6. Difference between correct and forward simulated moduli introducing a systematic error, $\epsilon^{\mathrm{s}}$, of \pm $1 \%$ in angle and height change measurements for (a) Layer 1 and (b) Layer 2.

From Figure 6 (a) it is observed that systematic errors in beam height causes the ratio between the correct and the inferred Layer 1 modulus to differ from unity. The ratio is in the range of 0.95-1.04 and 0.94-1.08 for Cases 1 and 2, respectively. Systematic errors in beam angle affect the ratio between correct and the inferred Layer 1 modulus to an even larger degree. This ratio is in the range of 0.17-0.71 and 0.56-1.39 for Cases 1 and 2, respectively.

A similar trend in the results may be observed in Figure 6 (b) for Layer 2. A systematic error of plus or minus one percent in height changes result in a non-unity ratio between the correct and the inferred modulus of Layer 2 within the range of 0.94-1.07 and 0.87-1.06 for Cases 1 and 2, respectively. For systematic errors in beam angle changes this ratio is in the range of $0.42-0.85$ and $0.56-1.96$ for Cases 1 and 2, respectively.

Overall, it appears that the interpretation method is sensitive to systematic beam angle changes and relatively insensitive to systematic beam height changes or to a systematic error in one of the laser distance readings. The high sensitivity to errors in angle change measurements is due to the geometry of the problem, i.e., a small error in $\Delta \theta_{i \rightarrow i-1}$ will result in large $d_{i}^{M}$ errors. It is also observed that there is no general trend in the results, indicating that the problem is case specific, i.e., influenced by the loading configuration, the position of the lasers, and pavement system considered. This means that the reliability of the device is not only a function of the device itself, given that also the tested pavement system influences the errors.

\subsection{Sensitivity to random errors}

This subsection investigates the influence of random errors, generically denoted as $\epsilon^{\mathrm{r}}$, on the interpretation method results. The investigation is based on a Monte Carlo approach, e.g. Hammersley and Handscomb (1964), which calls for re-executing the interpretation method many times over. A different set of input values is utilized in each execution-iteration, from which a new set of optimal moduli values are generated. By studying many such sets of optimal solutions it becomes possible to utilize statistical tools and quantify the effects that random measurement errors have on the 
interpretation method results.

The inputs for every Monte Carlo iteration were obtained based on Table 1, considering that they are not fixed, but are random variables obeying a symmetric triangular probability density function (PDF). The triangular density function is commonly used in Monte Carlo studies (see e.g. Newton and Smith (1992); Raftey (1991); Wilson (1982)) and selected herein as an easy way of estimating distribution parameters. The main advantage of the approach is that the variable of interest is restricted (i.e., has a definite upper and lower limit), bound by finite physical limits, and therefore avoiding unwanted/unphysical extreme values. The triangular distribution also enables the possibility to introduce skewed PDFs. Specifically, the values listed in Table 1 represent, in each case, the PDF mean. The range of parameter values about their mean was chosen based on the measurement capabilities of the most current Raptor device. These ranges are as follows: $\epsilon_{\mathrm{d}}^{\mathrm{r}}= \pm 25 \mu \mathrm{m}, \epsilon_{\Delta \theta_{i \rightarrow i-1}^{\mathrm{r}}}^{\mathrm{r}}= \pm 9 \cdot 10^{-6} \mathrm{rad}$, and $\epsilon_{\Delta h_{i \rightarrow i-1}}^{\mathrm{r}}= \pm 11$ $\mu \mathrm{m}$. The respective standard deviations are smaller, and can be obtained by simply dividing by $\sqrt{6}$.

The raw Monte Carlo results are presented in Figure 7 as cumulative distribution functions (CDFs). There are four charts in this Figure, referring to Cases 1 and 2, and to the inferred moduli for layers 1 and 2 . As can be seen, the abscissas are normalized w.r.t. the correct (target) moduli values, and the ordinates depict the cumulative distribution of the normalized moduli. 


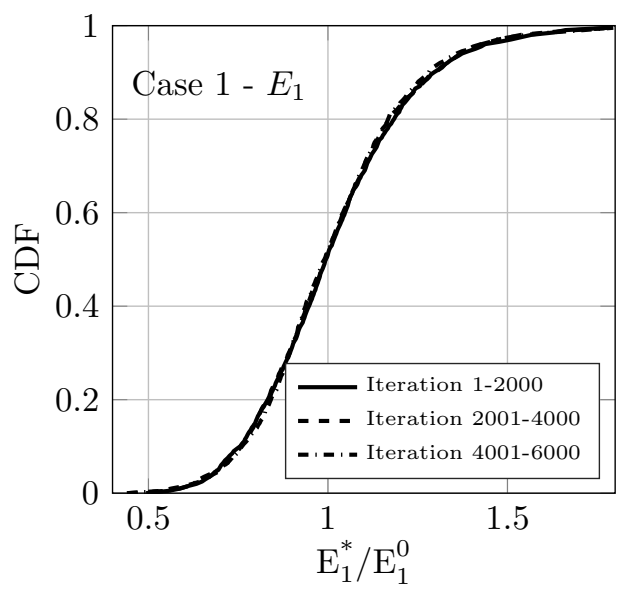

(a)

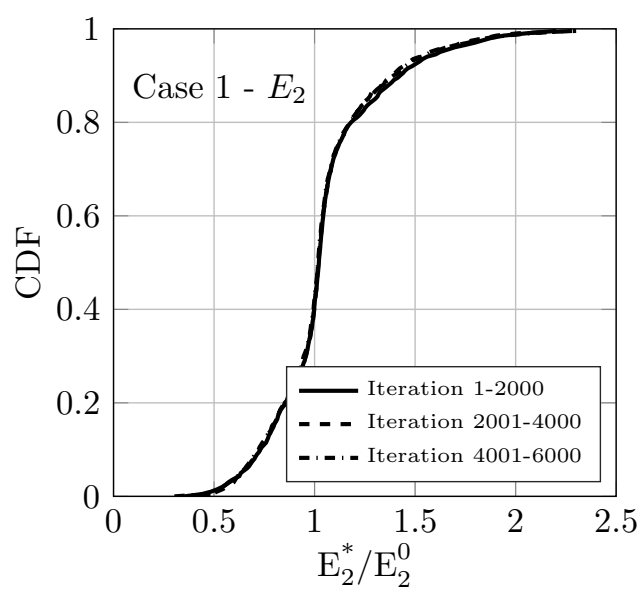

(c)

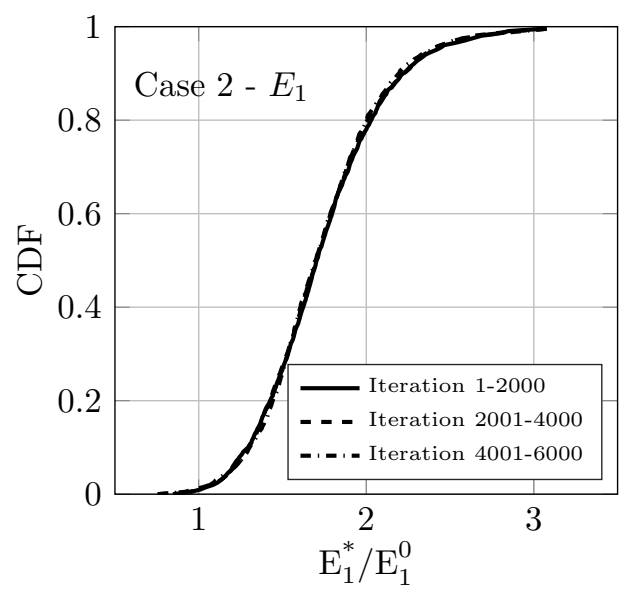

(b)

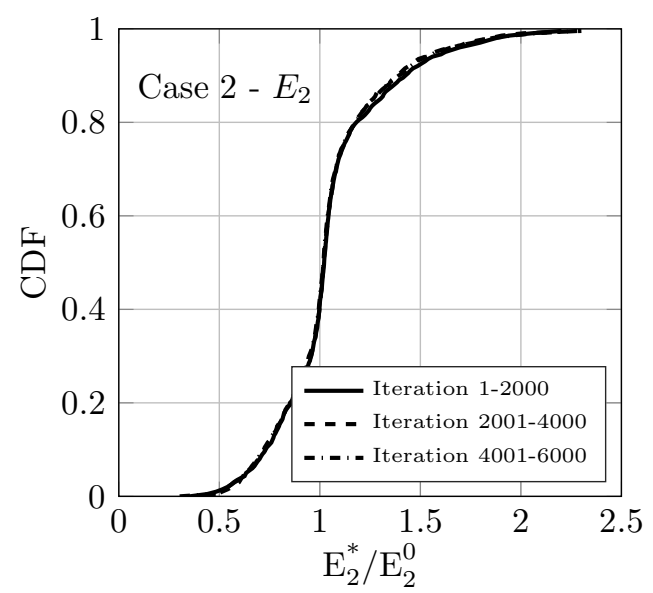

(d)

Figure 7. Monte Carlo simulation results after 2000 iterations presented as cumulative distribution functions (CDFs) for three different sets of random errors; (a) and (b) representing Layer 1 (i.e., the pavement structure), and (c) and (d) representing Layer 2 (i.e., the subgrade and deeper layers).

First and foremost, these charts were utilized to guide the choice for the number of appropriate Monte Carlo iterations. For this purpose each of the charts was regenerated three times with the same simulation effort. The resulting CDFs were then compared against one another; if they did not match then the chosen number of simulations was increased and new CDFs were generated. This procedure was repeated until the CDF triplets were graphically overlapping. As shown in Figure 7, the sufficient number of Monte Carlo iterations was found to be 2000 across all cases.

Next, the CDFs were analyzed for statistical content. This entailed fitting an analytic cumulative Beta distribution function, from which a PDF for each of the layer moduli was obtained. This approach was selected as it yields a smooth regular approximation to the unknown CDF from which the sample data was taken (see e.g., AbouRizk, Halpin, and Wilson (1994); Levenberg and Uzan (2004)). Moreover, the approach has the advantage that the PDF statistics can be calculated directly from analytical expressions. These PDFs are shown in Figure 8 (a)-(d) for the inferred moduli of Layer 1 (i.e., the pavement structure) and Layer 2 (i.e., the subgrade and deeper layers), respectively. 


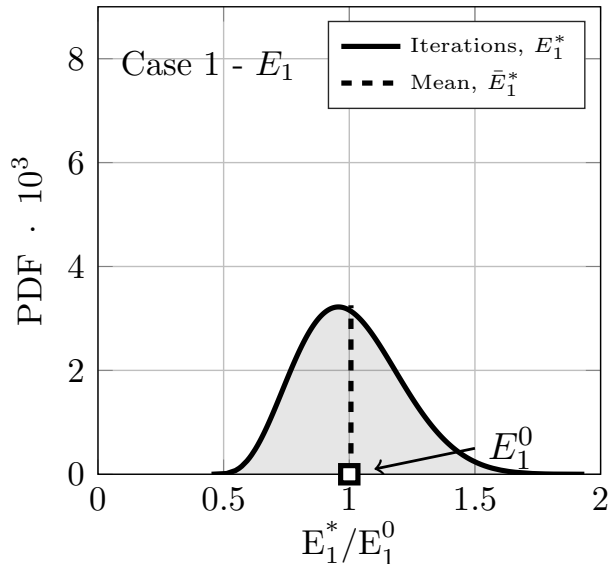

(a)

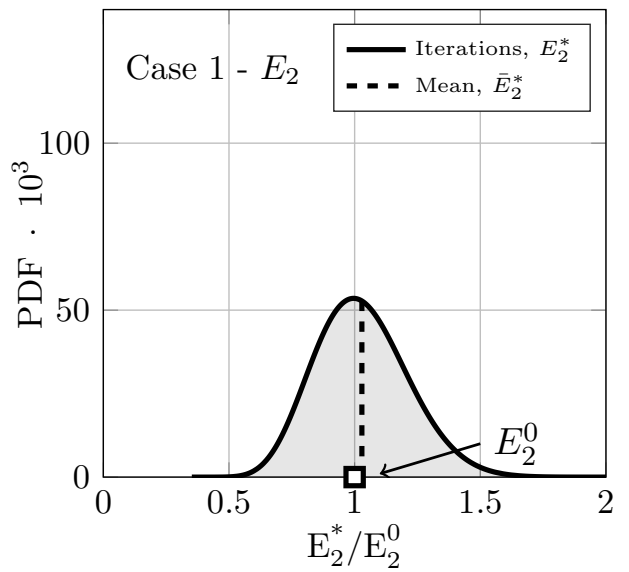

(c)

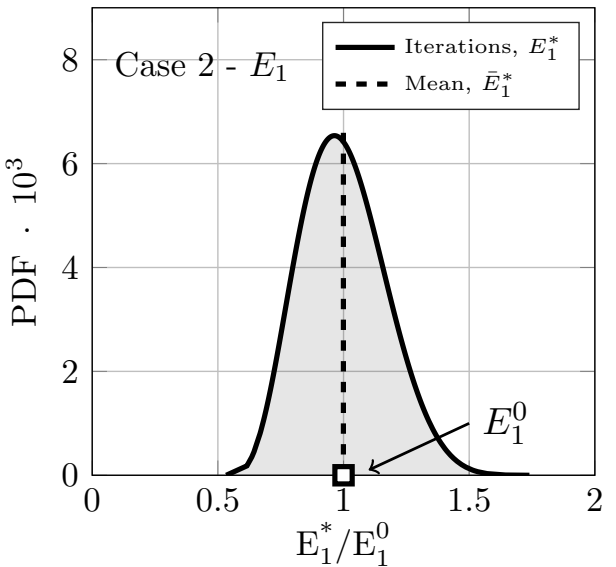

(b)

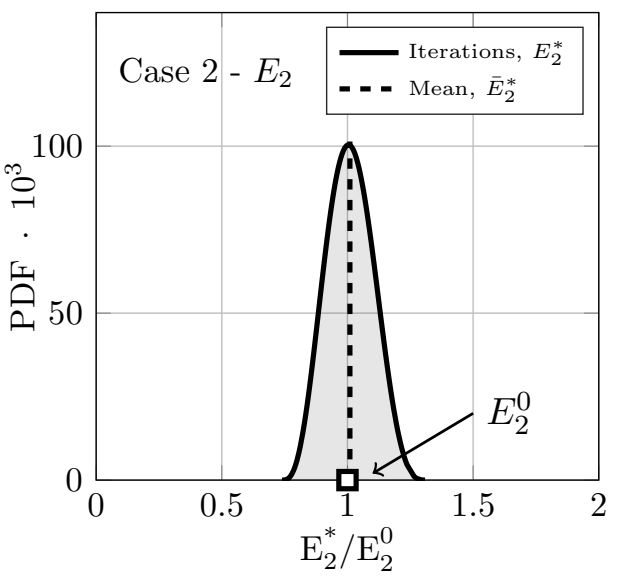

(d)

Figure 8. Beta probability density distribution after 2000 iterations for the inferred moduli; (a) and (b) representing Layer 1 (i.e., the pavement structure), and (c) and (d) representing Layer 2 (i.e., the subgrade and deeper layers). Predicted mean dashed line. Correct solution shown as square white mark.

From Figure 8 (a) and (b) it is observed that the PDF's for the inferred moduli of Layer 1 exhibit a slight positive skew, i.e., they have a tail to the right and a mode (peak) to the left of the mean. The difference between the predicted mean $\bar{E}_{1}^{*}$ and the correct $E_{1}^{0}$ is $+0.7 \%$ and $-0.01 \%$, for Case 1 and 2 , respectively.

From Figure 8 (c) and (d) it is observed that the PDF's for the inferred moduli of Layer 2 are positive skew, i.e., they have a tail to the right. The mean and mode are almost identical in both cases. The PDF of Case 2 resembles that of a normal distribution with a small coefficient of variation, indicating that the likelihood of determining the subgrade moduli and deeper layers, is high even with low data sampling rates. The difference between the predicted mean $\bar{E}_{2}^{*}$ and the correct $E_{2}^{0}$ is $+2.7 \%$ and $+0.7 \%$, for Case 1 and 2, respectively.

The results of the Monte Carlo analysis are listed in Table 3 which provides a summary of the resulting PDF statistics. 
Table 3. Summary of Monte Carlo analysis results in Figure 8

\begin{tabular}{ccccccc}
\hline \multirow{2}{*}{ Case } & \multirow{2}{*}{ Layer } & \multirow{2}{*}{ Iterations } & $\begin{array}{c}\text { Mean } \\
\bar{E}_{n}^{*} / E_{n}^{0}\end{array}$ & Mode & Coef. of variation & Skewness \\
\hline \multirow{2}{*}{1} & 1 & 2000 & 1.007 & 0.96 & 0.19 & 0.35 \\
& 2 & 2000 & 1.027 & 0.99 & 0.18 & 0.32 \\
\hline \multirow{2}{*}{2} & 1 & 2000 & 0.999 & 0.96 & 0.17 & 0.33 \\
& 2 & 2000 & 1.007 & 1.00 & 0.09 & 0.04 \\
\hline
\end{tabular}

\section{Conclusions and Recommendations}

This study focused on the Raptor MMP, and addressed three fundamental aspects needed for its eventual acceptance as a viable device for project-level pavement evaluation. The Raptors loading and measurement setup were first described. In general terms it consists of a beam equipped with several laser line profilers and also motion tracking sensors. The main outcome from this exposition is that the device is potentially capable, by means of patch matching (Figure 2), of tracking a point on the pavement surface while driving, and measure displacement information caused by its wheel configuration (Figure 1). An interpretation method was described next, capable of accepting measured Raptor data as input (Figure 3), and providing elastic properties for a layered pavement model. The third and final part of the work dealt with validating the interpretation method and quantifying the effects of measurement errors on the inferred moduli.

The interpretation method was initially operated with perfect (errorless) synthetic input data (Figure 4). This exercise showed strong convergence conditions to correct results (Figure 5). Thus, the overall concept passes the most basic validation step. Next, the interpretation method was operated with synthetic input data containing some systematic errors. This exercise revealed that inferred moduli may not be very sensitive to a single incorrect laser reading or wrong beam height-changes, but are rather sensitive to erroneous beam-angle changes (Figure 6). This finding emphasizes the importance of measuring angle changes as accurately and precisely as technology allows. Finally, by means of a Monte Carlo technique, the effects of random errors on the interpretation results were quantified. This evaluation (see Figures 7 and 8) revealed a range of about $\pm 50 \%$ of the correct value for individual interpretation results. It was also demonstrated that averaging the results of 2000 evaluations (for the same pavement system) yields a nearly unbiased estimate of the correct moduli. Considering a laser scan rate of $4000 \mathrm{~Hz}$, and a travel speed of $20 \mathrm{~m} / \mathrm{s}(72 \mathrm{~km} / \mathrm{h})$, obtaining 2000 evaluation points correspond to averaging over $10 \mathrm{~m}$ long pavement sections.

The present paper constitutes an initial development phase for a project-level method to interpret Raptor measurements. The next development phase involves application of the proposed interpretation method to real Raptor data, and validation of the results against an independent measurement system. One validation approach is to compare inferred moduli against FWD backcalculated results. Another possible validation approach is to instrument the tested section with different sensors, record the responses due to the passing Raptor, and then assess the pavement layer moduli independently from the sensor data (see e.g., Levenberg $(2013,2015)$ ).

Future efforts will also be expended on further improving the ability to track the 
sensor beam movements in-between patch detections. One idea in this connection is utilizing the laser readings themselves to help track the sensor beam movements. Additional modeling effort will be applied by considering a three-layered system, including viscoelasticity (rate and temperature dependence), accounting for fluctuating load levels (due to vehicle dynamics), and inclusion of sideway displacements of tracked points in the analysis. Addressing the latter point will require incorporation of sensor beam roll and yaw angle changes in the Raptor simulation module.

\section{Acknowledgements}

The authors would like to thank Innovation Fund Denmark [grant no. 5156-00004A] for financial support of the research presented in this paper. 


\section{References}

AbouRizk, S. M., Halpin, D. W., \& Wilson, J. R. (1994). Fitting beta distributions based on sample data. Journal of Construction Engineering and Management, 120(2), 288-305.

Andersen, S., Levenberg, E., \& Andersen, M. (2017). Inferring pavement layer properties from a moving measurement platform. Bearing Capacity of Roads, Railways and Airfields, 675-682.

Andersen, S., Levenberg, E., \& Andersen, M. B. (2018). Efficient reevaluation of surface displacements in a layered elastic half-space. International Journal of Pavement Engineering, $1-8$.

ASTM-D4694. (2015). Standard test method for deflections with a falling-weight-type impulse load device. ASTM International, West Conshohocken, PA.

ASTM-D4695. (2015). Standard guide for general pavement deflection measurements. ASTM International, West Conshohocken, PA.

Baltzer, S., Tønnesen, P., S., Busch, C., Gleerup, S., Holst, M. L., .. Thorup, C. (2017). Design of pavements and reinforcement layers. Vejregler - Danish Road Directorate, Ministry of Transport, Building and Housing.

Briggs, R. C., Johnson, R. F., Stubstad, R. N., \& Pierce, L. (1999). A comparison of the rolling weight deflectometer with the falling weight deflectometer. Astm Special Technical Publication(1375), 444-456.

Burmister, D. M. (1945). The general theory of stresses and displacements in layered systems. i. Journal of applied physics, 16(2), 89-94.

Chatti, K., Ji, Y., \& Harichandran, R. (2004). Dynamic time domain backcalculation of layer moduli, damping, and thicknesses in flexible pavements. Transportation research record, $1869(1), 106-116$.

Chou, Y., \& Lytton, R. L. (1991). Accuracy and consistency of backcalculated pavement layer moduli. Transportation Research Record, 1293, 72-85.

Elbagalati, O., Elseifi, M. A., Gaspard, K., \& Zhang, Z. (2017). Implementation of the structural condition index into the louisiana pavement management system based on rolling wheel deflectometer testing. Transportation Research Record, 2641(2641), 39-47.

Elbagalati, O., Mousa, M., Elseifi, M. A., Gaspard, K., \& Zhang, Z. (2018). Development of a methodology to backcalculate pavement layer moduli using the traffic speed deflectometer. Canadian Journal of Civil Engineering(ja).

Elseifi, M. A., Gaspard, K., Wilke, P. W., Zhang, Z., \& Hegab, A. (2015). Evaluation and validation of a model for predicting pavement structural number with rolling wheel deflectometer data. Transportation Research Record, 2525(2525), 13-19.

Flintsch, G., Katicha, S., Bryce, J., Ferne, B., Nell, S., \& Diefenderfer, B. (2013). Assessment of continuous pavement deflection measuring technologies (Tech. Rep.).

Garbowski, T., \& Pozarycki, A. (2017). Multi-level backcalculation algorithm for robust determination of pavement layers parameters. Inverse Problems in Science and Engineering, $25(5), 674-693$.

Goktepe, A. B., Agar, E., \& Lav, A. H. (2006). Advances in backcalculating the mechanical properties of flexible pavements. Advances in engineering software, 37(7), 421-431.

Graczyk, M., Zofka, A., Sudyka, J., \& Rafa, J. (2014). Analytical solution of pavement deflections and its application to the tsd measurements. In Arrb conference, 26th, 2014, sydney, new south wales, australia.

Gressin, J. (1982). The lacroix-lpc deflectograph. In Proceedings of a international symposium on bearing capacity of roads and airfields, volume 1, trondheim, held june 23-25, 1982.

Hammersley, J., \& Handscomb, D. (1964). Monte carlo methods. Methuen,.

Harr, M., \& Ng-A-Qui, N. (1977). Noncontact nondestructive determination of pavement deflection under moving loads. (Tech. Rep.). U.S. Department of Transportation, Systems Research and Development Service, Washington D.C. 2059.

Harr, M. E., \& Elton, D. J. (1983). Non-contact, non-destructive airport pavement profile, texture and deflection measurements. (Tech. Rep.). Purdue University Lafayette In School 
of Civil Engineering.

Herr, W. J., Hall, J. W., White, T. D., \& Johnson, W. (1995). Continuous deflection basin measurement and backcalculation under a rolling wheel load using scanning laser technology. Transportation Congress, Proceedings, 1, 600-611.

Horak, E., Hefer, A., Maina, J., \& Emery, S. (2015). Structural number determined with the falling weight deflectometer and used as benchmark methodology. Adv Eng Res, 5, 26-36.

Huang, Y. H. (2004). Pavement analysis and design., 2.

Ioannides, A. M., \& Khazanovich, L. (1998). General formulation for multilayered pavement systems. Journal of transportation engineering, 124(1), 82-90.

Irwin, L., Yang, W., \& Stubstad, R. (1989). Deflection reading accuracy and layer thickness accuracy in backcalculation of pavement layer moduli. In Nondestructive testing of pavements and backcalculation of moduli. ASTM International.

Irwin, L. H., \& Richter, C. A. (2005). History and development of u.s. procedures for falling weight deflectometer calibration. Transportation Research Record(1905), 67-72.

Katicha, S. W., Flintsch, G. W., \& Ferne, B. (2013). Optimal averaging and localized weak spot identification of traffic speed deflectometer measurements. Transportation Research Record, 2367(2367), 43-52.

Khazanovich, L., Tayabji, S. D., Darter, M. I., \& ERES Consultants, I. (2001). Backcalculation of layer parameters for ltpp test sections: Volume $i$ : Slab on elastic solid and slab on denseliquid foundation analysis of rigid pavements (Vol. RD-00-086). FHWA.

Kilareski, W. P., \& Anani, B. A. (1982). Evaluation of in-situ moduli and pavement life from deflection basins. 5th International Conference on the Structural Design of Asphalt Pavements, Proceedings, 1, 349-366.

Levenberg, E. (2013). Inverse analysis of viscoelastic pavement properties using data from embedded instrumentation. International Journal for Numerical and Analytical Methods in Geomechanics, 37(9), 1016-1033.

Levenberg, E. (2015). Backcalculation with an implanted inertial sensor. Transportation Research Record, 2525 (2525), 3-12.

Levenberg, E., Pettinari, M., Baltzer, S., \& Christensen, B. M. L. (2018). Comparing traffic speed deflectometer and falling weight deflectometer data. Transportation Research Record: Journal of the Transportation Research Board, 036119811876852.

Levenberg, E., \& Uzan, J. (2004). Quantifying the confidence levels of deformation measurements in asphalt concrete. Journal of Testing and Evaluation, 32(5), 1-365.

Madsen, S. S., \& Levenberg, E. (2018). Dynamic backcalculation with different load-time histories. Road Materials and Pavement Design, 19(6), 1314-1333.

Manoharan, S., Chai, G., Chowdhury, S., \& Golding, A. (2018). A study of the structural performance of flexible pavements using traffic speed deflectometer. Journal of Testing and Evaluation, 46(3), 1280-1289.

Mehta, Y., \& Roque, R. (2003). Evaluation of fwd data for determination of layer moduli of pavements. Journal of Materials in Civil Engineering, 15(1), 25-31.

Muller, W. B., \& Roberts, J. (2013). Revised approach to assessing traffic speed deflectometer data and field validation of deflection bowl predictions. International journal of pavement engineering, 14(4), 388-402.

Nasimifar, M., Siddharthan, R. S., Hajj, E. Y., \& Motamed, R. (2016). Investigation of 3d-move responses under traffic speed deflection devices (tsdds). In J. P. Aguiar-Moya, A. Vargas-Nordcbeck, F. Leiva-Villacorta, \& L. G. Loría-Salazar (Eds.), The roles of accelerated pavement testing in pavement sustainability (pp. 161-176). Cham: Springer International Publishing.

Nasimifar, M., Siddharthan, R. V., Rada, G. R., \& Nazarian, S. (2017). Dynamic analyses of traffic speed deflection devices. International Journal of Pavement Engineering, 18(5), 381-390.

Nasimifar, M., Thyagarajan, S., \& Sivaneswaran, N. (2017). Backcalculation of flexible pavement layer moduli from traffic speed deflectometer data. Transportation Research Record, 2641(2641), 66-74. 
Nelder, J. A., \& Mead, R. (1965). A simplex-method for function minimization. Computer Journal, 7(4), 308-313.

Newton, S., \& Smith, V. (1992). Methods of analysing risk exposure in the cost estimates of high quality offices. Construction management and economics, 10(5), 431-449.

Nondestructive testing of pavements and backcalculation of moduli (Vol. 1; Special publication). (1989). West Conshohocken, PA: ASTM International.

Nondestructive testing of pavements and backcalculation of moduli (Vol. 2; Special publication). (1994). West Conshohocken, PA: ASTM International.

Nondestructive testing of pavements and backcalculation of moduli (Vol. 3; Special publication). (2000). West Conshohocken, PA: ASTM International.

Pedersen, L. (2013). Viscoelastic modelling of road deflections for use with the traffic speed deflectometer (Unpublished doctoral dissertation).

Raftey, J. (1991). Principles of building economics: an introduction. Oxford Professional Books.

Sangghaleh, A., Pan, E., Green, R., Wang, R., Liu, X., \& Cai, Y. (2014). Backcalculation of pavement layer elastic modulus and thickness with measurement errors. International Journal of Pavement Engineering, 15(6), 521-531.

Siddharthan, R., Sebaaly, P. E., \& Javaregowda, M. (1996). Influence of statistical variation in falling weight deflectometers on pavement analysis. Transportation Research Record, 1377, $57-66$.

Simonin, J., Lièvre, D., Rasmussen, S., \& Hildebrand, G. (2005). Assessment of the danish high speed deflectograph in france. In Proc. 7th international conference on the bearing capacity of roads, railways and airfields, trondheim norway (Vol. 10).

Simonin, J.-M., Geffard, J.-L., \& Hornych, P. (2015). Performance of deflection measurement equipment and data interpretation in france. In International symposium non-destructive testing in civil engineering.(ndt-ce), berlin, alemania.

Sivaneswaran, N., Kramer, S. L., \& Mahoney, J. P. (1991). Advanced backcalculation using a nonlinear least squares optimization technique. Transportation research record(1293).

Stubstad, R., Irwin, L., Lukanen, E., \& Clevenson, M. (2000). It's 10 o'clock: Do you know where your sensors are? Transportation Research Record: Journal of the Transportation Research Board(1716), 10-19.

Ullidtz, P. (1987). Pavement analysis. developments in civil engineering. Elsevier.

Uzan, J. (1994). Advanced backcalculation techniques. In Nondestructive testing of pavements and backcalculation of moduli: Second volume. ASTM International.

Varma, S., Kutay, M., \& Levenberg, E. (2013). Viscoelastic genetic algorithm for inverse analysis of asphalt layer properties from falling weight deflections. Transportation Research Record: Journal of the Transportation Research Board(2369), 38-46.

Von Quintus, H., \& Simpson, A. (2002). Back-calculation of layer parameters for ltpp test sections, volume ii: Layered elastic analysis for flexible and rigid pavements (Vol. RD-01113.; Tech. Rep.).

Wilke, P. W., et al. (2015). Network level structural evaluation with rolling wheel deflectometer. In 9th international conference on managing pavement assets.

Wilson, A. (1982). Experiments in probabilistic cost modelling. Building Cost Techniques: New Directions (transactions of Building Cost Research Conference), 169-180.

Yi, J.-H., \& Mun, S. (2009). Backcalculating pavement structural properties using a neldermead simplex search. International Journal for Numerical and Analytical Methods in Geomechanics, 33(11), 1389-1406.

Zofka, A., Graczyk, M., \& Rafa, J. (2015). Qualitative evaluation of stochastic factors affecting the traffic speed deflectometer results. In Transportation research board 94th annual meeting washington dc.

Zofka, A., Sudyka, J., Maliszewski, M., Harasim, P., \& Sybilski, D. (2014). Alternative approach for interpreting traffic speed deflectometer results. Transportation Research Record: Journal of the Transportation Research Board(2457), 12-18. 